\title{
Diversidad de aves en áreas en proceso de regeneración natural degradas por minería a cielo abierto de oro y platino en Condoto, Chocó, Colombia
}

\section{Diversity of birds in areas undergoing natural regeneration degraded by gold and platinum mining in Condoto, Chocó, Colombia}

\section{Eric Yair Cuesta-Ríos ${ }^{1}$, Ismael Palacios Palacios ${ }^{2}$, Kelly Jhoana Moreno Mena², Jessica Katherine Morales Asprilla²}

\section{Resumen}

Con el objetivo de determinar la diversidad de la comunidad de aves asociadas con áreas en proceso de regeneración natural, degradas por minería a cielo abierto de oro y platino en Condoto, Chocó, Colombia, se desarrollaron muestreos, utilizando métodos como los censos y la captura con redes de niebla, entre las 06:00 hasta las 18:00 horas. Se registraron 662 individuos, distribuidos en 10 órdenes, 22 familias, 49 géneros y 62 especies. El orden Passeriformes tuvo el mayor número de familias y especies. A nivel de familias Thraupidae (S:11), Tyrannidae (S:9) y Trochilidae (S:9), fueron las familias más ricas. La curva de saturación de especies exhibió un patrón diferente en cada uno de los escenarios, donde a nivel general se registró el 73\% de la riqueza estimada, factor que fue atribuido a las características que exhibió cada área muestreada. En relación con la diversidad, el escenario 1 registró la mayor diversidad $\left(H^{\prime}=3,4\right)$, igualmente la mayor equidad de pielou $(0,90)$ ymenordominancia $(D=0,04)$. No se registraron especies amenazas. Por último, se registraron tres especies migratorias, lo que ratifica el bosque pluvial tropical como un centro importante de migraciones para las aves, donde gracias a la variedad de ecosistemas se propician escenarios para albergar variedad de aves que llegan hasta estos ecosistemas a cumplir con sus procesos postreproductivos.

Palabras clave: Avifauna, Bosques fragmentados, Gremios tróficos, Minería, Resiliencia, Región del San Juan.

\begin{abstract}
With the objective of determining the diversity of the bird community associated with the areas undergoing natural regeneration, the mining zones and the open gold and platinum sky in Condo
\end{abstract}

Investigador Asociado II, Instituto de Investigaciones Ambientales del Pacífico (IIAP), Grupo Investigación Conocimiento, Manejo y Conservación de los Ecosistemas del Chocó Biogeográfico, Quibdó, Colombia.

e-mail: cuestarios@gmail.com

Biólogo con Énfasis en Recursos Naturales, Universidad Tecnológica del Chocó, Quibdó, Colombia. e-mail: kejhomome1309@gmail.com 


\section{Bioetnia Volumen 14, 2017}

to, Chocó, Colombia, sampling was carried out, using methods such as censuses and captured with fog networks, between 06:00 and 18:00 hours. There were 662 individuals, distributed in 10 orders, 22 families, 49 genera and 62 species. The order Passeriformes had the largest number of families and species. At the family level Thraupidae (S:11), Tyrannidae (S:9) and Trochilidae (S:9) were the richest families. The species saturation curve exhibited a different pattern in each of the scenarios, where $73 \%$ of the estimated richness was recorded, a factor that was attributed to the characteristics exhibited by each sampled area. In relation to diversity scenario 1 , the greatest diversity $\left(H^{\prime}=3.4\right)$ was recorded, as well as the greater skin fairness (0.90) and lower dominance $(D=0.04)$. No threat species were recorded. Finally, three migratory species were registered, which ratifies the tropical rainforest as an important migratory center for birds, where, thanks to the variety of ecosystems, scenarios are provided to house a variety of birds that reach these ecosystems to comply with their Postreproductive processes.

Keywords: Avifauna, Fragmented forests, Mining, Resilience, San Juan Region, Trophic guilds.

\section{Introducción}

Los bosques tropicales son conocidos por ser ecosistemas altamente dinámicos, debido a la influencia de una serie de factores y procesos ambientales (Scatena 2002), en donde las perturbaciones naturales forman una parte integral de esta dinámica (García-Montiel et al. 2002). Sin embargo, a pesar de los constantes cambios como consecuencia de su inherente condición dinámica, la presencia humana deja huellas significativas sobre los ecosistemas boscosos (García-Montiel et al. 2002), sobre todo aquella asociada con las actividades productivas que trae como consecuencia la remoción de la cobertura vegetal en extensas áreas.

En relación con lo anterior, claro ejemplo de ello son los bosques de las selvas del Chocó, que gracias a actividades antropogénicas como la minería y la tala, han provocado la transformación, remoción y fragmentación de los bosques, lo que ha producido la pérdida de la diversidad animal (Cuesta-Ríos et al. 2007, IIAP 2012), que resulta alarmante si se observa la tasa de remoción de cobertura boscosa en los últimos años, donde la merma de bosquees cada vez mayor, perdiéndose alrededor de 6'488.940 ha de bosques, y el Chocó es uno de los departamentos donde esta ha sido mayor (IDEAM 2014), no solo por la eliminación física de hábitats, sino una combinación entre este proceso, la cacería y otros factores lo que puede estar produciendo esa pérdida de la diversidad animal.

Con la disminución de la cobertura vegetal, los grupos animales, en particular las aves, se ven fuertemente afectadas, porque muchas de sus especies requieren de conectividad del paisaje y de las estructuras básicas que ofrece la vegetación para el hábitat y la alimentación de muchos grupos biológicos.

En el caso particular de la minería a cielo abierto de oro y platino, Vargas et al. (2010), señala que esta actividad causa numerosos impactos de largo alcance en espacio y tiempo para todos los componentes ambientales, porque se contaminan las fuentes hídricas y fragmentan otros ecosistemas, además de generar cambios en la estructura y función de las comunidades bióticas; este fenómeno trae consigo total afectación de las dinámicas poblacionales de las comunidades animales propias de un lugar y sus zonas aledañas, dada la migración de ciertas especies que entran a competir por espacio y alimento con las que ya habitaban en dicho ecosistema.

Los impactos de la minería a cielo abierto de oro y platino, se sienten fuertemente en los grupos faunísticos terrestres en especial grupos de hábitos estrictamente arbóreos como ranas arborícolas y 


\section{Diversidad de aves en áreas en proceso de regeneración natural. EY Cuesta-Ríos et al.}

lagartos, mamíferos como los perezosos que son de poca movilidad y naturaleza "tímida", primates y aves, que a pesar de presentar alta movilidad, dependen exclusivamente del recurso vegetal, el cual les brinda disponibilidad de hábitat, que es vital en los procesos de alimentación y reproducción de estos grupos.

Las aves son organismos de gran importancia en los bosques húmedos tropicales convirtiéndose en los grupos más importantes en la dinámica ecológica y supervivencia de los mismos que son considerados como los mejores dispersores de semillas en términos de cantidad, distancia y transporte (Molinari 1993, Ortiz-Pulido et al. 2000) y por ser dispersores legítimos de la mayoría de las especies de semillas que ellos ingieren (Fleming y Sosa 1994), adquiriendo un rol fundamental en la regeneración de los bosques nativos.

Las aves poseen un sinnúmero de ventajas sobre el aporte al proceso de regeneración de los bosques, especialmente los tropicales y en algunos subtropicales, debido a que su permanencia en estos ecosistemas es durante casi todo el año, contribuyendo de manera constante al proceso de restauración; además, permiten medir su aporte en el proceso, porque además de ser diurnas y fáciles de observar, son capaces de responder rápidamente a las modificaciones ambientales debido a su movilidad (Brosset et al. 1996), también son valiosas en el estudio del impacto de las alteraciones producidas por la acción del hombre, a corto y largo plazo (Fenton et al. 1992).

En este sentido y con el propósito de entender la situación actual de la avifauna y su dinámica en diversas áreas entre las que se incluyen áreas alteradas por minería en el Chocó, se han venido ejecutando diversos estudios, que dan cuenta de lo que se tiene, en cuanto a la diversidad de las aves de esta región, sus problemáticas y la importancia que tienen para dinámica ecológica de los bosques y los diferentes servicios ambientales que prestan. En este orden de ideas, a nivel departamental se ha venido destacando una serie de trabajos realizados sobre las comunidades de aves, los cuales han aumentado el conocimiento de este grupo faunístico, pero los esfuerzos han sido insuficientes, porque aún existen enormes vacíos de información relacionados con los impactos de la minería a cielo abierto en el Chocó, hectáreas destruidas, minas activas y aún más sobre la capacidad de recuperación de cada uno de los componentes afectados. En lo relacionado con las aves, la información es escaza y aislada, porque la mayor parte de los trabajos han sido orientados al estudio de la biodiversidad ornitológica y dejando de lado aspectos ecológicos y poblacionales de las especies; pero el propósito es seguir investigando para tener un conocimiento más amplio del que se tiene, por lo cual en la presente investigación se pretendió determinar la diversidad de aves presentes en áreas proceso de regeneración natural degradas por minería a cielo abierto de oro y platino en Condoto, Chocó, Colombia, información con la cual se pretende contribuir a generar información importante para comprender la dinámica de este grupo taxonómico en áreas alteradas por diversos factores de origen antrópico.

Área de estudio. El trabajo se desarrolló en el corregimiento de Jigualito, municipio de Condoto, que geográficamente se localiza a los $5^{\circ} 06^{\prime} 01^{\prime \prime}$ norte y $76^{\circ} 32^{\prime} 44^{\prime}$ " oeste, a $70 \mathrm{msnm}$ (Figura 1), dentro de la ecoregión del Chocó Biogeográfico, que geopolíticamente en Colombia es denominada costa pacífica. Pertenece a la zona de vida de bosque húmedo tropical (bh-T). Presenta una temperatura megatermal entre $25^{\circ} \mathrm{C}$ y $28^{\circ} \mathrm{C}$, una humedad relativa que la ubica dentro de las zonas con balance hídrico perhúmedo a superhúmedo $(86 \%)$ y una precipitación zonas de pluviosidad moderadamente alta $(8.000 \mathrm{~mm})$ Poveda et al. (2004).

Los sitios de muestreo fueron seleccionadas de acuerdo con una investigación previa, realizada por el IIAP (2012), ubicados en el municipio de Condoto, en las coordenadas geográficas $\mathrm{N} 05^{\circ} 02^{\prime}$ 


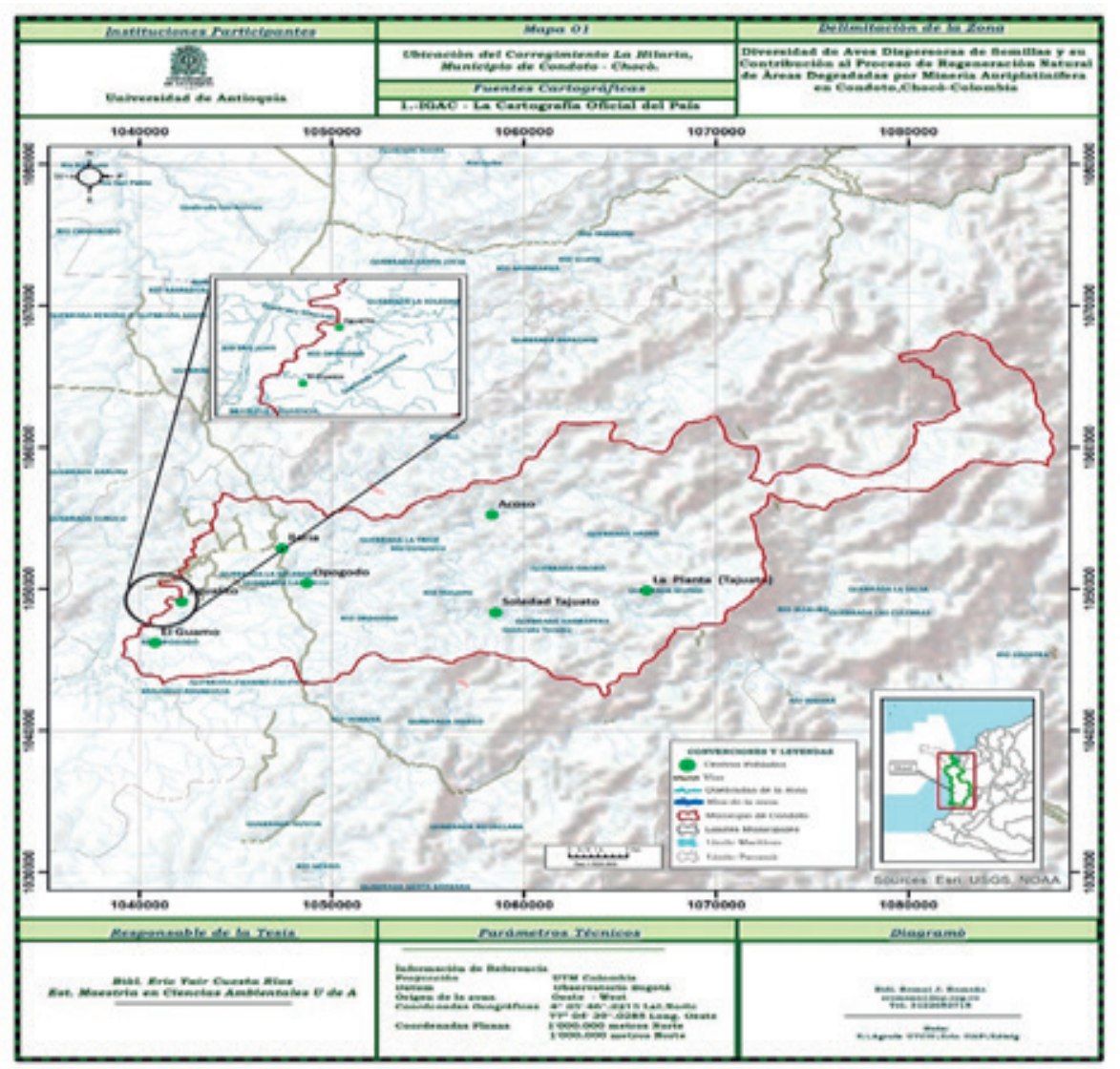

Figura 1. Ubicación geográfica del área de estudio.

$45^{\prime \prime} \mathrm{N}$ y $76^{\circ} 42^{\prime} 20.8^{\prime \prime} \mathrm{O}$. Se caracterizan por ser bosques que fueron fuertemente intervenidos por actividad minera a cielo abierto para la extracción de oro y platino. Las áreas escogidas cumplen con las siguientes características:

Escenario 1 o Bosque de referencia. Correspondió a un bosque que no ha sido intervenido con actividad minera, el cual evidencia un importante estado de conservación. Igualmente se observó un gran potencial para la ocurrencia de especies faunísticas de comportamiento crítico, raras o asociadas con hábitats maduros. Su arquitectura y estructura vegetal presentó dos estratos bien diferenciados; un nivel arbustivo que estuvo representado por arbustos entre 3 y 7 metros, mientras que el arbóreo con árboles entre 8 y 30 metros, con una importante presencia de palmas, que proporcionan alimento y sustrato para aquellas especies de vertebrados de comportamientos arborícolas o que incluyen este recurso en sus comportamientos tróficos.

Escenario 2. Bosque entre 30 y 50 años, de cese de la actividad y donde existe un avanzado proceso de regeneración natural, exhibiendo un bosque donde se distinguen claramente tres estratos: herbáceo, arbustivo y arbóreo, dominados por Mimosaceas, Caesalpiniaceae, y Melastomateceae, entre otras.

Escenario 3. Bosque entre 5-15 años: corresponde a un área intervenida recientemente afectada por la actividad minera y ha tenido alrededor de 6 años para recuperarse, evidenciándose los primeros procesos de sucesión. En la cual se observó un importante número de plantas que fueron dispersadas por aves, donde se destacan Vismia baccifera y Miconia sp. El suelo aún desnudo, con poca hojarasca. 


\section{Diversidad de aves en áreas en proceso de regeneración natural. EY Cuesta-Ríos et al.}

\section{Métodos}

El trabajo de campo tuvo una duración de seis meses, tiempo durante el cual se realizaron tres visitas por mes a las zonas objeto de estudio con una duración de cinco días, donde se realizaron muestreo de 12 horas diarias, entre 06:00-18:00,y se registraron ejemplares empleando dos métodos de muestreo:

Capturas con redes. Por cada sitio de muestreo se instalaron seis redes de niebla $(12 \times 3 \mathrm{~m})$, por escenario, en horarios que van desde las 06:0018:00 horas, siguiendo lo propuesto por Bibby y Burgess (2000). A los especímenes capturados se les tomaron registros fotográficos y sus respectivos datos de campo, luego fueron almacenados en bolsas de tela, marcados con un corte en una o varias plumas caudales con el propósito de evitar tomar datos de aves recapturadas y posteriormente se liberaron.

Censos desde puntos de radio fijo. Bajo esta técnica, se tuvo en cuenta todas las aves que se vieron y escucharon en un tiempo determinado y preferiblemente antes de las 10 am cuando están más activas. Esta metodología se realizó ad libitum, para completar el listado taxonómico. Se establecieron nueve puntos de radio fijo (tres por zona), en el centro de un círculo imaginario de 5 metros de radio y se realizaron conteos de aves durante 10 minutos con la ayuda de binoculares (10 x 40); entre cada punto se tomó una distancia mínima $100 \mathrm{~m}$.

La determinación taxonómica se realizó mediante la revisión de guías ilustradas de campo de McMullan etal. (2011) y clasificadas siguiendo la propuesta de Remsen et al. (2016). Finalmente, se identificaron las especies amenazadas de extinción de acuerdo con Renjifo et al. (2014), endémicas de acuerdo con Chaparro-Herrera et al. (2013) y migratorias de acuerdo con Naranjo et al. (2012).

Para el análisis de la composición y estructura de la comunidad de aves, se calculó la riqueza como el número de especies por sitio de muestreo.
Los rangos de abundancia que se determinaron se obtuvieron según los criterios descritos en Villareal et al. (2004). Además, se elaboraron y graficaron curvas de diversidad-dominancia, que expresan la representatividad de especies en los tres escenarios evaluados. También se estimó la representatividad del muestreo a través de las curvas de acumulación de especies, generada mediante el programa Stimates (versión 6.0), utilizando estimadores no paramétricos (Chao 2 y Bootstrap).

Para el análisis estructural de la comunidad de aves se aplicaron índices de diversidad de Shannon-Wiener (H'), equidad de Pielou (J') y el índice de dominancia (D) en cada escenario muestreado, según como se describe en Magurran (1988), Moreno (2001) y Villareal et al. (2004); también se realizó la prueba Kruskal-Wallis para evaluar las diferencias de las abundancias, riquezas y diversidad en los escenarios de muestreo. Estos análisis se realizaron en el programa estadístico PAST 1.22 (Hammer et al. 2001). Para cada escenario, se construyó una matriz de especies $v s$. unidades de muestreo, se realizó un análisis de complementaridad y similaridad de Jaccard a nivel especifico y de Bray Curtis como una medida de la diferencia entre las abundancias de cada especie presentes en el área de estudio.

\section{Resultados y discusión}

Composición de la avifauna asociada con las áreas. Se registraron 662 individuos: 509 por censos y 153 capturados en redes. El total de individuos estuvieron distribuidos en 10 órdenes, 22 familias, 49 géneros y 62 especies (Anexo). El orden Passeriformes tuvo el mayor número de familias (Figura 2a) y de especies (Figura 2b).

Los resultados aquí expuestos guardan relación de las características del ambiente con la ornitofauna presente; muestra a los Passeriformes como el orden más representativo en cuanto a número de familias y de especies, presentando un patrón 

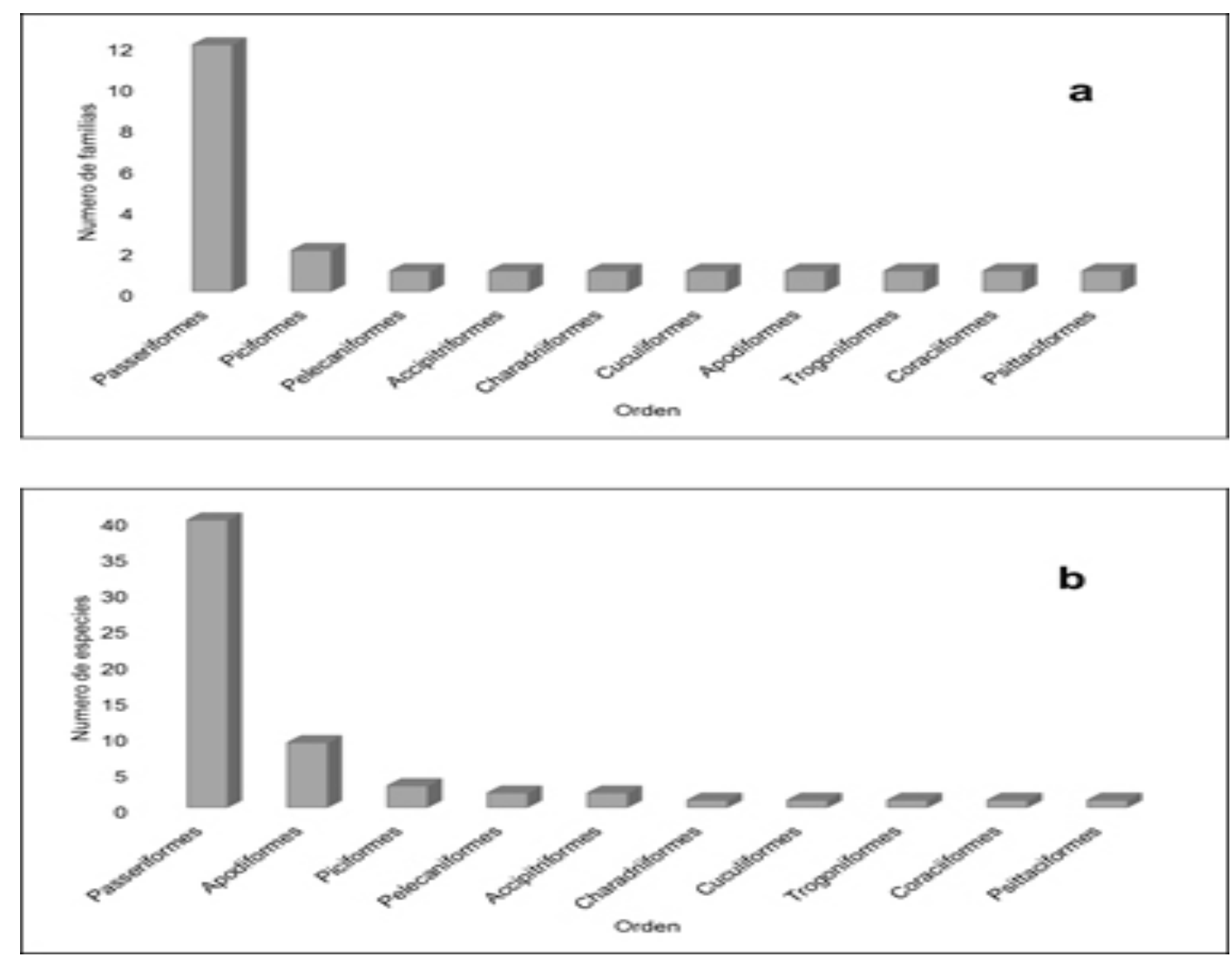

Figura 2. Distribución de las familias (a) y riqueza específica (b) de los órdenes en la comunidad de aves en áreas en proceso de regeneración natural degradas por minería a cielo abierto de oro y platino en Condoto, Chocó, Colombia.

similar con otros trabajos en los bosques húmedos del Chocó (Machado y Peña 2000, Córdoba y Cuesta 2003, Caicedo y Serna 2005, Ríos et al. 2007, Mosquera et al. 2008, Ramírez 2009, Perea 2014), donde este orden fue el más diverso.

La representatividad de los Passeriformes obedece quizás a la capacidad que presentan estas aves de adaptarse a una variada gama de ambientes, gracias a su alta radiación adaptativa que les ha permito ser el grupo más exitoso en los ecosistemas tropicales (Guallar et al. 2009), apreciación que es compartida por Tabilo-Valdivieso (2006), quien además aduce que los Passeriformes son el grupo más especializado dentro de las aves, además presenta una excelente radiación adaptativa en hábitats tropicales, lo cual les permite acoplarse fácilmente a ecosistemas aunque estén modificados y/o transformados.
Las familias Thraupidae (S:11), Tyrannidae (S:9) y Trochilidae (S:9), presentaron la mayor riqueza, estando por encima de familias como Thamnophilidae, Furnariidae y Emberizidae, que presentaron máximo tres especies (Figura 3).

Estos datos tienden a exhibir un patrón similar a los resultados obtenido por otros investigadores en la selva pluvial central del Chocó; por ejemplo, Ríos et al. (2007), en áreas de expansión urbana en el municipio de Quibdó, Mosquera et al. (2008), en dos tipos de cobertura vegetal en Pacurita, municipio de Quibdó, Ramírez (2009), en sistemas agroforestales en municipio de Lloró, Borja et al. (2009) en tres ambientes paisajísticos del municipio de Nuquí, e IIAP (2012), en zonas degradadas por la actividad minera a cielo abierto en los municipios de Tadó y Condoto, registran a las familias en mención, como las de 


\section{Diversidad de aves en áreas en proceso de regeneración natural. EY Cuesta-Ríos et al.}

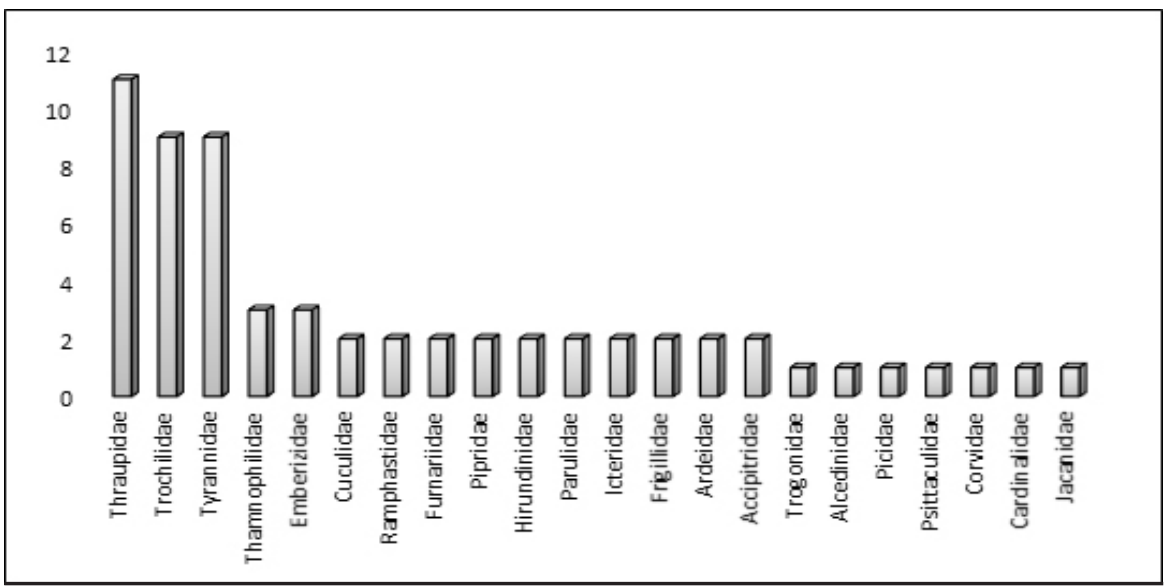

Figura 3. Distribución de la riqueza específica de las familias en la comunidad de aves en áreas en proceso de regeneración natural degradas por minería a cielo abierto de oro y platino en Condoto, Chocó, Colombia.

mejor representación a nivel específico en sus respetivos trabajos. En relación con lo anterior la composición taxonómica que exhibe el presente trabajo, no es novedad en las selvas húmedas del Chocó Biogeográfico; por lo general, son familias frecuentes en estos ambientes y sus ecosistemas asociados, obedeciendo quizás a características como su plasticidad ecológica, que les permite colonizar y adaptarse a diferentes condiciones climáticas y biofísicas que presentan los bosques húmedos de esta región, lo que se ve reflejado el su abundancia y diversidad específica.

En cuanto a las familias Thraupidae y Tyrannidae, esta representación se debe a que algunas especies de estas familias, son más abundantes en bosques húmedos tropicales, donde se alimentan casi por completo de una fuente muy variada y fácil de encontrar como los insectos, frutas de los árboles y arbustos que crecen en la selva; además, les ofrece una gran variedad de ecosistemas y hábitats que satisfacen a la comunidad de aves (Hilty y Brown 2001). En relación con esto, si se hace un análisis de la historia de vida de traupidos, tyrannidos registrados en la zona, se encuentra que en su mayoría presentan conductas ecológicas diversas, las cuales le permiten desplegar grandes adaptaciones a diferentes ecosistemas con altos niveles de antropización, por ejemplo, en áreas disturbadas por minería aluvial, en los cuales habitan y exhiben toda su flexibilidad a los diferentes cambios que estos ambientes presentan. Al respecto, autores como Daily et al. (2001), señalan que muchas de las aves que componen estas familias, se pueden adaptar con facilidad a hábitats altamente modificados. La capacidad de utilizar estos hábitats probablemente se deba a adaptaciones obtenidas por presiones de selección, lo que hace que aquellas especies que están en constantes presiones tengan mayor capacidad de tolerar y sobrevivir en estos paisajes modificados (Harris y Pimm 2004).

En relación con la familia Trochilidae, se debe a la constante oferta habitacional y trófica disponible en el área, pues debido a sus hábitos generalistas, encuentran un árbol donde anidar y una planta en proceso de floración o en su efecto una colonia de insectos que le permiten suplir sus demandas alimenticias. Esto fue evidenciado en el páramo, donde la presencia de familias cuyas características florales (flores tubulares, gamopétalas, con alta producción de néctar y colores vistosos), que incluyen las familias Rubiaceae, Ericaceae, Genneriaceae, que permiten a miembros de esta familia de aves visitarlas y proveerse de néctar.

Abundancia de las especies asociadas con los escenarios de estudio. Las especies más abun- 


\section{Bioetnia Volumen 14, 2017}

dantes con porcentajes superiores al 5\% fueron: Tachyphonus delatrii (pi:-1,15), Crotophaga major (pi:-1,18), Manacus manacus (pi:1,19) y Tangara larvata (pi:-1,21). La distribución de las abundancias de las especies fue marcada entre los tres escenarios. Solo 9,6\% de las especies presentaron abundancias superiores o iguales a los 30 individuos; $23 \%$ de las especies presentaron abundancias intermedias (15-25) y un poco más del $60 \%$ de las especies presentaron una abundancia inferior a 15 individuos, lo que refleja la gran cantidad de especies raras encontradas en esta investigación.

En E1 se obtuvo la mayor representatividad de las especies; M. manacus (pi:-1,04), T. larvata (pi=-1,11), E. flavogaster, $R$. dimidiatus, T. delatrii $(p i=-1,27)$. Estos escenarios registraron 20 especies exclusivas (Sporagraxanthogastra, Euphonia xanthogaster, Parula pitiayumi, Saltator grossus, Saltatormaximus, Saltator striatipectus, Tachyphonus rufus, Mitrospingus casiinii, Tyrannus Tyrannus, Pyrocephalus rubianus, Myizetetes granadensis, Dendrocincla fuliginosa, Glyphorynchus spirurus, Contopus cinereus, Hylophylax naevioides, Myrmotherula axillaris, Ramphastos brevis, Ramphastos swainsonii, Androdon aequatorialis, Florisuga mellivora).

En el E2, Tachyphonus delatrii ( $p i=-0,91)$, Tangara larvata ( $p i=-1,14)$, y Amazilia tzacatl ( $p i=-1,17)$, fueron las más abundantes, mientras que Accipiter superciliosus, Accipiter striatus, Threnetes ruckeri, Trogon chionurus, Colaptes punctigula, Myibius barbatus, Cyanocorax affinis, Piranga rubra y Psarocolius wagleri, fueron exclusivas para este escenario.

Para el E3, Volatina jacarina ( $i=-0,70)$, Crotophaga major ( $p i=-0,79)$ y Sporophila corvina $(p i=-0,83)$. En este escenario se encontraron seis especies exclusivas entre las que hay cuatro acuáticas o dependientes por el medio acuático (Butorides striata, Egretta thula, Jacana jacana, Chloroceryle amazona), Volatinajacarina y Spo- rophila corvina. En el caso de las aves acuáticas en estos ecosistemas alterados se debe quizás a la alta oferta de humedales artificiales que deja la actividad minera que hace que estas especies aprovechen de manera importante estos lugares porque muchos de sus ecosistemas naturales están sujetos a diversas alteraciones por actividades antrópicas entre estas la minería.

Al respecto Sebastián-González et al. (2010), señala que la pérdida de zonas húmedas naturales, algunas de las construcciones de origen antrópico han comenzado a ser usadas como hábitat alternativo para las especies dependientes de los humedales. La mayoría de estos humedales artificiales ofrecen un hábitat aprovechable por las aves en alguna época del año o en todo momento (Múrias et al. 2002, Elphick y Oring 2003).

Salinas, balsas de piscifactorías, lagunas mineras, graveras, campos de arroz o balsas de riego ofrecen un hábitat alternativo a aves, así como a anfibios, plantas o invertebrados (Knutson et al. 2004). De hecho, el Convenio Ramsar ha empezado a incluir humedales artificiales en su lista de Humedales de Importancia Internacional. Sin embargo, parece ser que, en comparación con los humedales artificiales, los humedales naturales son, en general, capaces de sustentar mayores y más diversas comunidades de animales y plantas (Ma et al. 2004)

Por escenarios, numéricamente se evidencian diferencias ligeramente marcadas en relación con la riqueza específica y número de individuos (Tabla 1), donde el mayor valor se encontró en el E1 o escenario de referencia, con 45 especies y 296 individuos, superando al E2, donde se registraron 35 especies y 250 individuos. En el E3 solo se registraron 15 especies y 116 individuos. A pesar de que existió cierta variación entre las abundancias y riquezas de los tres escenarios, los análisis mostraron que las diferencias no fueron estadísticamente significativas $(\mathrm{K}-\mathrm{W}, \mathrm{p}=0.40)$ y $(\mathrm{K}-\mathrm{W}, \mathrm{p}=0.47)$, respectivamente. 
Tabla 1. Representatividad de los diferentes taxones de la avifauna asociada con áreas en proceso de regeneración natural degradas por minería a cielo abierto de oro y platino en Condoto, Chocó, Colombia

\begin{tabular}{lrcc}
\hline Taxón & E1* $^{*}$ & E2 (30-50) & E3 (5-15) \\
\hline Familias & 14 & 14 & 8 \\
Especies & 45 & 35 & 15 \\
Individuos & 296 & 250 & 116 \\
\hline
\end{tabular}

* Referencia

Riqueza estimada de la avifauna asociada con áreas en proceso de regeneración natural. Los resultados de los estimadores muestran que el esfuerzo de muestreo no fue significativo para registrar un porcentaje representativo de la comunidad de aves en los escenarios 1 y 2 , donde se obtuvo $58 \%$ y $66 \%$ respectivamente de la riqueza esperada (Tabla 2). Mientras que para el E3, el muestreo fue significativo, logrando registrar $88 \%$ de la riqueza de este escenario. Cabe resaltar que este escenario ofrece poca oferta tanto trófica como de hábitat, que las aves que se registraron aquí generalmente están de paso. Estos datos son coincidentes con lo que exhibe el ecosistema, porque áreas con mayor perturbación antrópica se encuentran asociadas sobre todo con ambientes con poca disponibilidad de recursos, mientras que las áreas con mayor tiempo de recuperación, suelen estar asociadas con la cobertura herbácea, arbustiva y de dosel, lo que promueven disponibilidad de recursos tróficos, hábitat y microhábitats.
Las curvas de saturación de especies para los escenarios 1 y 2 , muestran que es necesario seguir realizando muestreos para conocer la riqueza absoluta, porque si se emplea un esfuerzo mayor las posibilidades de seguir registrando especies incrementaría. Mientras que para elE3, el muestreo fue representativo; según lo consignado en Villareal et al. (2004), para que un muestreo se considere representativo deben registrarse al menos $86 \%$ de las especies, es decir que el muestreo alcanzó una representatividad suficiente (Figura 4).

Estos comportamientos de la curva se asemejan a los resultados de Guevara y Rodríguez (2014), donde en tres áreas con diferentes estados de la sucesión ecológica, las curvas mostraron esta misma tendencia, comportamiento que se asocia con la disponibilidad de recursos de cada escenario, porque a mayor oferta mayor puede ser el número de especies.

Diversidad alfa y beta de avifauna dispersoras de semillas. La diversidad medida con métodos directos exhibe una diversidad alta en términos generales, siendo mayor en el E1 $(\mathrm{H}=3,4)$, escenario que mostró mayor equitabilidad $(0,90)$ y menor dominancia $(D=0,04)$, lo que señala que en este escenario lo recursos están mucho mejor repartidos para soportar una avifauna con comportamientos tróficos y habitacionales heterogéneos. Al realizar un análisis de T-student para comparar estos escenarios con base en el valor de Shannon Winner indicó que existen diferencia significativa entre ellas (T-student:2,92; p: 0,04) (Tabla 3).

El hecho de que el escenario de referencia

Tabla 2. Riqueza esperada y porcentaje de representatividad del muestreo según el estimador de Chao 2 para la avifauna asociada con áreas en proceso de regeneración natural degradas por minería a cielo abierto de oro y platino en Condoto, Chocó, Colombia

\begin{tabular}{lccc}
\hline Escenario & Riqueza & Riqueza esperada (Chao 2) & \% de representatividad del muestreo \\
\hline E1 & 45 & 77 & 58 \\
E2 & 35 & 53 & 66 \\
E3 & 15 & 17 & 88 \\
Toda el área & 62 & 84 & 73 \\
\hline
\end{tabular}




\section{Bioetnia Volumen 14, 2017}
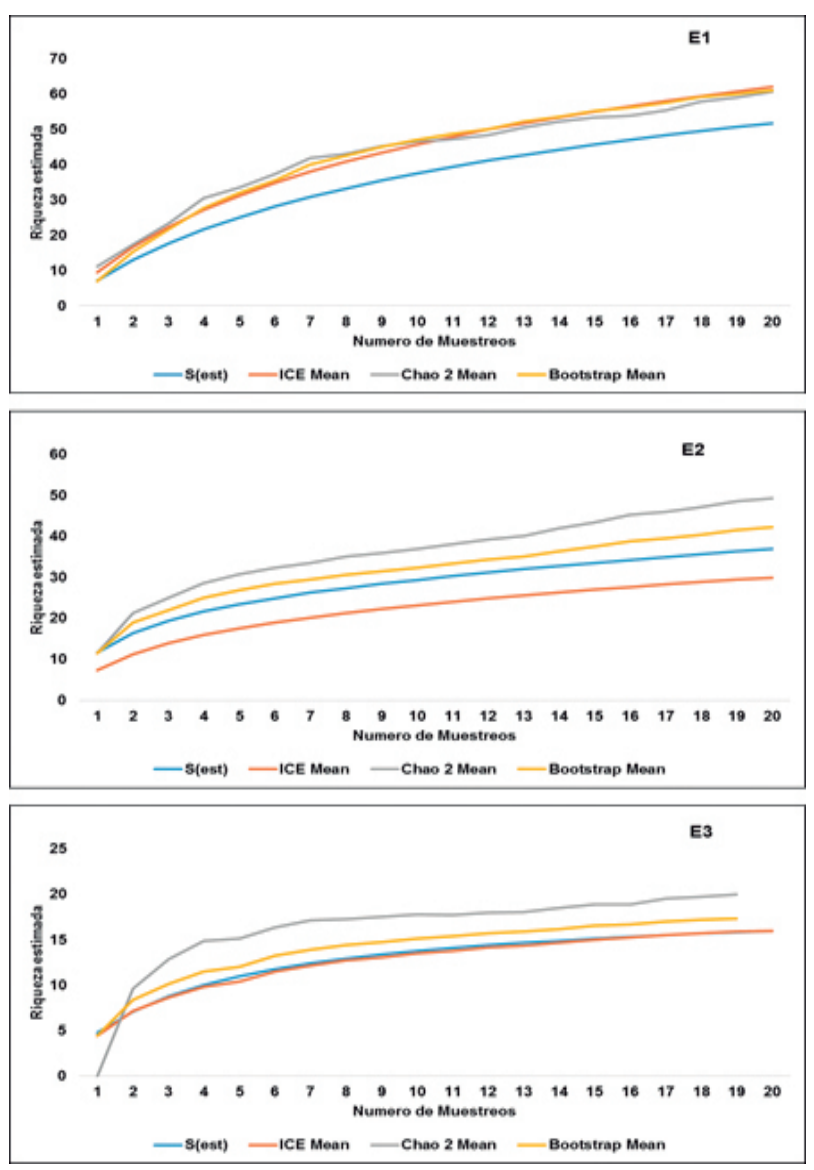

Figura 4. Curvas de saturación de especies para medir la riqueza de la avifauna asociada con áreas en proceso de regeneración natural degradas por minería a cielo abierto de oro y platino en Condoto, Chocó, Colombia.

haya sido el que presentó la mayor diversidad, obedece a las características exhibidas por la zona, dada las particularidades de esta para albergar una avifauna especial, marcada por sus propios requerimientos de tróficos y de hábitat.

En este escenario, se evidenció un buen estado de conservación, el cual es ratificado por la presencia de grupos claves y en la estructura básica de las formaciones tipológicas vegetales o de paisaje que presenta, lo que permite albergar un sin número de aves, que dependen de óptimas condiciones del ambiente, por sus altos requerimientos ecológicos, tales como Psittacidos, Ramphastidos, Thamnophilidos y Furnariidos, que según las apreciaciones de Rodríguez-Mahecha y Hernández-Camacho (2002) y Gilardi y Munn (1998), son indicadores biológicos de la salud del ambiente, calidad del hábitat y conectividad del paisaje; además, cumplen con procesos ecológicos fundamentales en el mantenimiento de la buenas condiciones ambientales de los bosques como la dispersión de semillas.

El E3 muestra un panorama muy diferente en cuanto a diversidad, donde se registró un valor de $(H=2,3)$; estos valores responden a las características que presentan, donde los disturbios son notables y se ha perdido en gran parte la estructura arbórea y el bosque apenas se está comenzando a recuperar, lo que se refleja directamente en la baja ocurrencia de aves, registrando especies comunes de área urbanas o de ecosistemas jóvenes. Al respecto, Lusk (1996) señala que la biodiversidad está determinada en función de las perturbaciones, las cuales repercuten positiva o negativamente dependiendo de la intervención; en este caso, las repercusiones son negativas, evidenciado en una baja riqueza ornitológica y con gran presencia de especies generalistas como: Volatina jacarina, Sporophila corvina, Crotophaga ani, Crotophaga major, Myizetetes cayanensis, estas especies están asociadas con áreas disturbadas, las cuales fueron abundantes en este escenario.

El porcentaje de similitud expresado por los índices Jaccard para la riqueza y Bray-Curtis para la abundancia (Figura 5), muestran que existe un bajo grado de semejanza entre los tres escenarios evaluados. Para la riqueza (Jaccard) existe solo $18 \%$ de similaridad entre E3 con E2 y E1, y 21\% en cuanto a la abundancia (Bray-Curtis), entre E3 con E2 y E1.

Especies de interés ecológico especial. No se registraron especies amenazadas. Según la estacionalidad, se logró registrar, tres especies migratorias, entre las que tenemos a Hirundo rustica, Piranga rubra, Parkesia noveboracensis, todas de hábitos migratorios boreales (MB), dato que ratifica el bosque pluvial tropical como un centro importante de migraciones para las aves a 


\section{Diversidad de aves en áreas en proceso de regeneración natural. EY Cuesta-Ríos et al.}

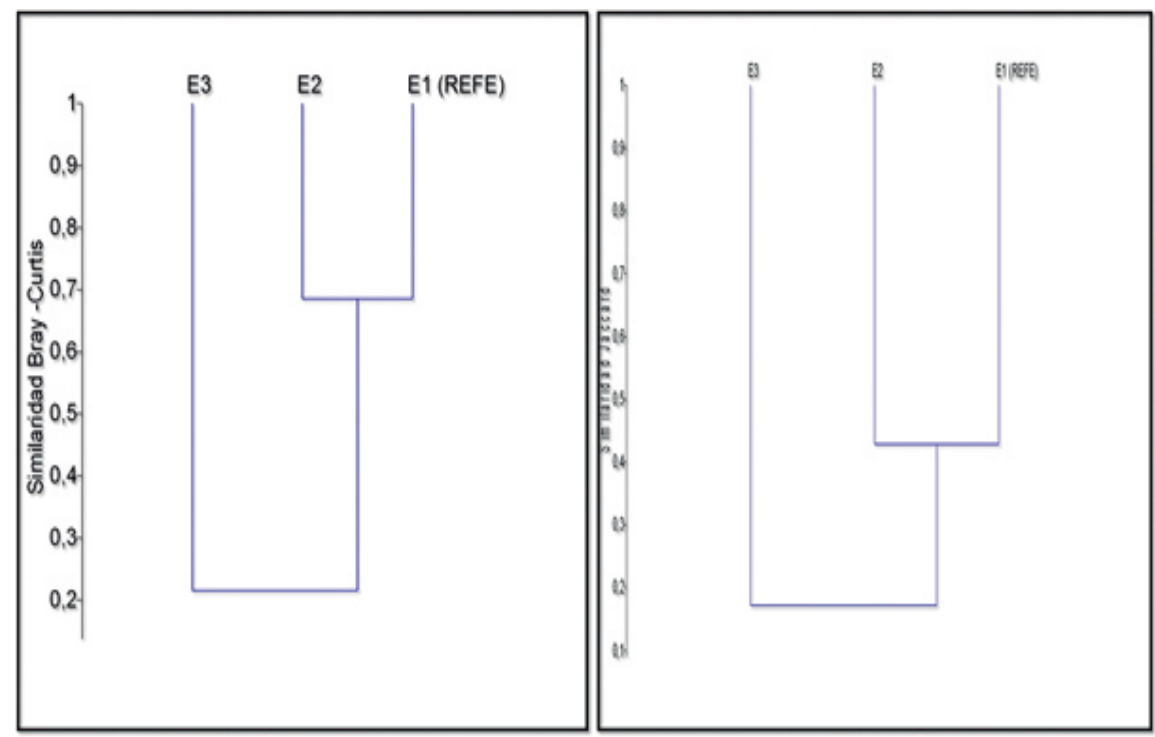

Figura 5. Análisis Cluster (Jaccard y Bray-Curtis) para la avifauna asociada con áreas en proceso de regeneración natural degradas por minería a cielo abierto de oro y platino en Condoto, Chocó, Colombia.

Tabla 3. Índices para conocer la diversidad de aves asociada con áreas en proceso de regeneración natural degradas por minería a cielo abierto de oro y platino en Condoto, Chocó, Colombia

\begin{tabular}{lrrrr}
\hline Índice & E1 & E2 & E3 & T-Studend \\
\hline Riqueza (S) & 45 & 35 & 15 & \\
Abundancia (n) & 296 & 250 & 116 & \\
Shannon Winner (H') & 3,5 & 3,2 & 2,3 & 2,92 \\
Dominancia (D) & 0,04 & 0,05 & 0,12 & \\
Pielou (J') & 0,91 & 0,90 & 0,86 & \\
\hline
\end{tabular}

pesar de estar altamente alterados, donde gracias a las áreas en estado avanzado de recuperación, las lagunas artificiales producto de minería y algunos mosaicos entre vegetación secundaria y cultivos de pancoger, crean una variedad de ecosistemas, que albergan aves que llegan hasta estos ecosistemas a cumplir con sus procesos postreproductivos.

La cifra de tres especies migratorias esta levemente por debajo de lo registrado por Ríos et al. (2007) y Mosquera et al. (2008), quienes en sus trabajos en dos tipos de cobertura vegetal en el corregimiento de Pacurita, municipio de Quibdó y cabecera municipal de Quibdó respectivamente, registraron la presencia de siete especies migratorias. Al respecto, Ocampo-Peñuela (2010) señala que la persistencia de las poblaciones de aves en bosques tropicales depende de la oferta ambiental de estos y sus ecosistemas asociados, los cuales permiten a las aves suplir sus requerimientos habitacionales y nutricionales durante el período de migración.

Gremios tróficos. A nivel general se registraron siete gremios tróficos, donde los frugívoros e insectívoros fueron los más representativos en los tres escenarios. En el E1, los frugívoros registraron $47 \%$, los insectívoros $31 \%$, nectarívoros $15 \%$, el reto de los gremios presentó $0 \%$ (Figura 6). En E2 los frugívoros con $31 \%$ e insectívoros con $23 \%$ fueron los mejor representados. E1 E3, mostró que frugívoros con $40 \%$ y piscívoros con $27 \%$ dominó esta zona.

El dominio de los insectívoros y frugívoros, se pudo dar quizás, por las características del componente vegetal, porque los estados secundarios en la sucesión vegetal y en los bosques maduros, existe una producción relativamente constante de frutos y alberga una buena cantidad de insectos, 


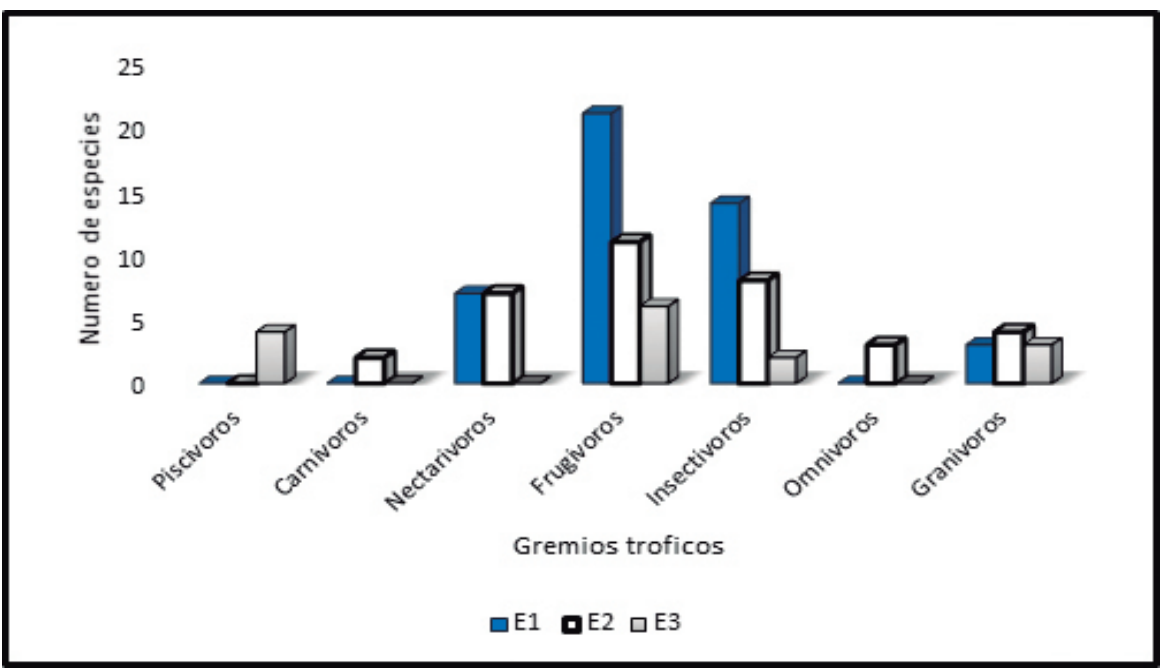

Figura 6. Representatividad de los gremios tróficos de la comunidad de aves asociada con áreas en proceso de regeneración natural degradas por minería a cielo abierto de oro y platino en Condoto, Chocó, Colombia.

aspecto que en gran medida resulta ser saludable para los ecosistema, porque la permanencia de las aves cumpliendo funciones ecológicas claves para el mantenimiento de la integridad de los ambientes naturales, siendo las principales el proceso de dispersión de semilla y polinización. Esto ratificado por (Moreno y Morales 2016) quienes manifiestan que los bosques tropicales, atraen gran cantidad de aves, principalmente las que se alimentan de insectos y frutos, que a la postre juegan un papel importante en la regeneración del bosque, porque en el ejercicio de sus funciones ecológicas determinan, junto con otros factores, la estructura y composición de los futuros bosques.

\section{Literatura citada}

Bibby CJ, Burgess ND, Hill DA, Mustoe S. 2000. Bird Census Techniques. $2^{\text {nd }}$ ed. San Diego: Academic Press Limited.

Borja JA, Gómez FL, Rengifo JT. 2012. Diversidad de aves presentes en tres ambientes paisajísticos del municipio de Nuquí, Chocó. Investigación, Biodiversidad y Desarrollo.; 31 (1): 31-42. Disponible en: https://issuu. com/rengifojt/docs/aves de nuqu

Brosset A, Charles-Dominique P, Cockle A, Cosson JF, Masson D. 1996. Bat communities and deforestation in French Guiana. Can J Zool. 74 (11): 1974-82.
Disponible en: http://www.nrcresearchpress.com/doi/ abs/10.1139/z96-224\#.WrTkfejOWUk

Caicedo M, Serna N. 2005. Aves Passeriformes en la cuenca hidrografía del río Cabí. Chocó, Colombia. Trabajo de grado. Quibdó: Universidad Tecnológica del Chocó, Facultad de Ciencias Básicas, Programa de Biología con Énfasis en Recursos Naturales; 58 pp.

Córdoba ML, Cuesta A. 2003. Ecología y estructura taxonómica de la comunidad de aves Passeriformes presentes en dos zonas de bosques pluvial tropical (bp-T). (Trabajo de grado). Programa de Biología con Énfasis en Recursos Naturales, Facultad de Ciencias Básicas, Universidad Tecnológica del Chocó, Quibdó, Chocó; 69 pp.

Cuesta-Ríos EY, Valencia-Mazo JD, Jiménez-Ortega AM. 2007. Aprovechamiento de los vertebrados terrestres por una comunidad humana en los bosques tropicales (Tutunendo, Chocó, Colombia). Revista Institucional Universidad Tecnológica del Chocó. 26 (2): 37-43. Disponible en: http://revistas.utch.edu.co/ojs5/index. php/revinvestigacion/article/view/478

Chaparro-Herrera S, Echeverry-Galvis MA, Córdoba-Córdoba S, Sua-Becerra A. 2013. Listado actualizado de las aves endémicas y casi-endémicas de Colombia. Biota Colomb. 14 (2): 113-50. Disponible en: http://revistas. humboldt.org.co/index.php/biota/article/view/289

Daily GC, Ehrlich PR, Sánchez-Azofeifa GA. 2001. Countryside biogeography: use of human-dominated habitats by the avifauna of southern Costa Rica. Ecol Appl. 11: 1-13. Disponible en: https://esajournals.onlinelibrary. wiley.com/doi/pdf/10.1890/1051-0761\%282001\%290 


\section{Diversidad de aves en áreas en proceso de regeneración natural. EY Cuesta-Ríos et al.}

11\%5B0001\%3ACBUOHD \%5D2.0.CO\%3B2

Elphick CS, Oring LW. 2003. Conservation implications of flooding rice fields on winter waterbird communities. Agr Ecosyst Environ. 94 (1): 17-29. Disponible en: https://www.sciencedirect.com/science/article/pii/ S0167880902000221

Fenton MB,Acharya L,AudetD, Hickey MBC, Merriman C, Obrist MK, et al. 1992. Phyllostomid bats (Chiroptera: Phyllostomidae) as indicators of habitat disruption in the newotropics. Biotropica. 24 (3): 440-6. Disponible en: $\underline{\text { https://www.jstor.org/stable/2388615?seq=1\#page }}$ scan tab contents

Fleming TH, Sosa VJ. 1994. Effects of nectarivorous and frugivorous mammals on the reproductive success of plants. J Mammal. 75: 845- 51.

García-Montiel DC, Melillo JM, Steudler PA, Neill, C, Feigl BJ, Cerri, CC. 2002. Relación entre la $\mathrm{N}_{2} \mathrm{O}$ y $\mathrm{CO}_{2}$ emisiones procedentes de la cuenca amazónica. Geophys Res Letters. 29 (6): 14-1-14-3. Disponible en: https://agupubs.onlinelibrary.wiley.com/doi/ abs/10.1029/2001GL013830

Gilardi JD, Munn CA. 1998. Patterns of activity, flocking, and habitat use in parrots of the Peruvian Amazon. Condor. 100 (4): 641-53. Disponible en: https://www.jstor. org/stable/1369745?seq=1\#page scan tab contents

Guallar S, Santana E, Contreras S, Verdugo H, Gallés A. 2009. Passeriformes del occidente de México: morfrometría, datación y sexado. Barcelona: Museu de Ciències Naturals, Institut Botànic de Barcelona; 491pp. Disponible en: http://monografies.museucienciesjournals.cat/files/MMCN-volum-5-Passeriformes-Occidente-de-M $\%$ C3\%A9xico-BR-color.pdf

Guevara L, RodríguezE. 2014. Composición y estructura de la comunidad de aves asociadas a áreas con diferentes grados de perturbación antrópica en la cabecera municipal de Atrato, Chocó, Colombia. Tesis de pregrado. Quibdó: Programa de Biología con énfasis en Recursos Naturales, Facultad de Ciencias Básicas, Universidad Tecnológica del Chocó; 57 pp.

Hammer O, Harper DAT, Ryan, PD. 2001. PAST: Palaeontological Statistics Software Package for Education and Data Analysis, ver. 1.88. Disponible en: http:// palaeo-electronica.org/2001 1/past/issue1 01.htm

Harris GM, Pimm SL. 2004. Bird species' tolerance of secondary forest habitats and its effects on extinction. Conserv Biol. 18 (6): 1607-16. Disponible en: $\underline{\text { https:// }}$ onlinelibrary.wiley.com/doi/full/10.1111/j.15231739.2004.00336.x-i1

Hilty SL, Brown WL. 2001. Guía de aves de Colombia. New Jersey: Princeton University Press; 1040 pp.

Instituto de Hidrología, Meteorología y Estudios Ambientales (IDEAM). 2014. Leyenda nacional de coberturas de la tierra. Metodología CORINE Land Cover adaptada para Colombia. Escala 1:100.000. Bogotá: Instituto de Hidrología, Meteorología y Estudios Ambientales.

Instituto de Investigaciones Ambientales del Pacífico(IIAP). 2012. Protocolo de restauración ecológica de áreas degradadas por minería a cielo abierto de oro y platino en el Chocó Biogeográfico. Informe final. Quibdó: IIAP; 84 pp. Disponible en: https://siatpc.iiap.org.co/docs/ avances/protocolo_de_restauracion_ecologico.pdf

Knutson MG, Richardson WB, Reineke DM, Gray BR, Parmelee JR, Weick SE. 2004. Agricultural ponds support amphibian populations. Ecol Appl. 14 (3): 669-84.

Lusk CH. 1996. Gradient analysis and disturbance history of temperate rain forest of coast range summit plateau, Valdivia, Chile. Rev Chil Hist Nat. 69: 401-11. Disponible en: http://rchn.biologiachile.cl/pdfs/1996/3/ Lusk 1996.pdf

Ma Z, LI B, Zhao B, Ping K, Tang S, Chen J. 2004. Are artificial wetlands good alternatives to natural wetlands for waterbirds? A case study on Chongming Island, China. Biodiv Conserv. 13 (2): 333-50. Disponible en: https://link.springer.com/article/10.1023/B:BIOC.0000006502.96131.59

Machado M, Peña G. 2000. Estructura numérica de la comunidad de aves del orden Passeriformes en dos bosques con diferentes grados de intervención antrópica en los corregimientos de Salero y San Francisco de Icho. Tesis de pregrado. Quibdó: Programa de Biología con énfasis en Recursos Naturales, Facultad de Ciencias Básicas, Universidad Tecnológica del Chocó; 40 pp.

Magurran AE. 1988. Ecological diversity and ist mesaurement. New Jersy: Princeton University Press; 192 pp.

McMullan M, Donegan TM, Quevedo A. 2011. Guía de campo de las aves de Colombia. Bogotá: Proaves; $225 \mathrm{pp}$.

Molinari J. 1993. El mutualismo entre frugívoros y plantas en las selvas tropicales: aspectos paleobiológicos, autoecologías, papel comunitario. Acta BiolVenez. 14 (4): 1-44. Disponible en: https://www.researchgate. net/profile/Jesus Molinari/publication/259756190 El mutualismo entre frugivoros y plantas en las_selvas tropicales_aspectos paleobiologicos autoecologias papel comunitario/links/0deec52d9d515af771000000.pdf

Mosquera LA, Hurtado Y, Rengifo JT. 2008. Diversidad aves en dos tipos cobertura vegetal en Pacurita, municipio de Quibdó, Chocó, Colombia. Revista Institucional Universidad Tecnológica del Chocó: Investigación, Biodiversidad y Desarrollo 2008, 27 (2): 231-39. Disponible en: http://revistas.utch.edu.co/ojs5/index. $\mathrm{php} /$ revinvestigacion/article/view/456/469

Moreno CE. 2001. Métodos para medir la biodiversidad. 


\section{Bioetnia Volumen 14, 2017}

M\&T Manuales Tesis SEA. Zaragoza: Programa Iberoamericano de Ciencia y Tecnología para el Desarrollo (CYTED), ORCYT/UNESCO \& SEA; 86 pp. Disponible en: http://entomologia.rediris.es/sea/ manytes/metodos.pdf

Moreno KJ, Morales JK. 2016. Aves paseriformes dispersoras de semillas y la legitimidad de su dispersión en áreas disturbada por minería a cielo abierto con diferentes estados de sucesión, en Condoto, Chocó, Colombia. Trabajo de grado para optar por el título de biólogo. Quibdó: Universidad Tecnológica del Chocó; 63 pp.

Múrias T, Cabral JA, Lopes RJ, Marques JC, Goss-Custard J. 2002. Use of traditional salines by waders in the Mondego estuary (Portugal): a conservation perspective. Ardeola. 49 (2): 223-40. Disponible en: https://www. researchgate.net/publication/237715085 Use of traditional salines by waders in the Mondego estuary Portugal A conservation perspective

Naranjo LG, Amaya JD, Eusse-González D, Cifuentes-Sarmiento Y(eds.). 2012. Guía de las especies migratorias de la biodiversidad en Colombia. Vol. 1. Aves. Bogotá: Ministerio de Ambiente y Desarrollo Sostenible, WWF Colombia; 710 pp. Disponible en: http://www. horizonteverde.org.co/attachments/article/19/PDFmigratorias aves 42 final.pdf

Ortiz-Pulido R, Laborde J, Guevara S. 2000. Frugivoría por aves en un paisaje fragmentado: consecuencias en la dispersión de semillas. Biotropica. 32 (3): 473-88. Disponible en: https://onlinelibrary.wiley.com/doi/ abs/10.1111/j.1744-7429.2000.tb00494.x

Poveda G, Mesa O, Salazar LF, Arias PA, Moreno HA, Vieira SC, et al. 2004. The diurnal cycle of precipitation in the tropical Andes of Colombia. Monthly Weather Rev. 133 (1): 228-40. Disponible en: https://journals. ametsoc.org/doi/abs/10.1175/MWR-2853.1

Ocampo- Peñuela NO. 2010. El fenómeno de la migración en aves: una mirada desde la Orinoquia. Orinoquia. 14 (2): 188-200. Disponible en: http://www.scielo.org.co/ pdf/rori/v14n2/v14n2a09.pdf

Perea Y. 2014. Diversidad alfa y beta de la avifauna en zonas de bosque pluvial Tropical de la estación ambiental del Alto San Juan en el municipio de Tadó, Chocó, Colombia. Trabajo de grado como requisito parcial para optar al título de Biólogo con Énfasis en Recursos Naturales. Quibdó: Universidad Tecnológica del Chocó; 53 pp.

Ramírez F. 2009. Aves asociadas a sistemas agroforestales en el Centro Multipropósito de Investigaciones de la
Universidad Tecnológica del Chocó. Trabajo de grado. Quibdó: Facultad de Ciencias Básicas, Programa de Biología con Énfasis en Recursos Naturales, Universidad Tecnológica del Chocó; 61 pp.

Remsen JVJr, Cadena CD, Jaramillo A, Nores M, Pacheco JF, Pérez-Emán J, et al. 2016. A classification of the bird species of South America. American Ornithologists'Union. Dispsonible en: http://www.museum.lsu. edu/ Remsen/SACCBaseline.html

Renjifo LM, Gómez MF, Velásquez-Tibatá J, Amaya-Villarreal AM, Kattan GH, Amaya-Espinel JD, et al. 2014. Libro rojo de aves de Colombia. Volumen 1. Bosques húmedos de los Andes y la costa pacífica. Bogotá: Pontificia Universidad Javeriana e Instituto Alexander von Humboldt; 465 pp. Disponible en: http://repository. humboldt.org.co/handle/20.500.11761/32545

Ríos O, García IH, Rengifo JT. 2007. Inventario de aves passeriformes en áreas de expansión urbanas presente en el municipio de Quibdó, Chocó, Colombia. Revista Institucional Universidad Tecnológica del Chocó. 26 (1): 79-89. Disponible en: http://revistas.utch.edu.co/ ojs5/index.php/revinvestigacion/article/view/469/481

Rodríguez-Mahecha JV, Hernández-Camacho JH. 2002. Loros de Colombia. Bogotá Desarrollo Nacional para la Conservación de las Aves de Colombia; 478 pp.

Sebastián-González E, Sánchez-Zapata JA, Botella F. 2010. Agricultural ponds as alternative habitat for waterbirds: spatial and temporal patterns of abundance and management strategies. Eur J Wildlife Res. 56(1): 11-20. Disponible en: https://hal.archives-ouvertes.fr/ hal-00535232

Scatena F. 2002. El bosque neotropical desde una perspectiva jerárquica. pp. 23-42. En: Guariguata MR; Kattan GH (eds). Ecología y conservación de Bosques Neotropicales. Cartago: Ediciones LUR; 693 pp.

Tabilo-Valdivieso E. 2006. El Censo Neotropical de Aves 2004. Global Series $N^{o}$ 17. Buenos Aires: Wetlands Internacional; pp. 83-6.

Vargas L, Quesada Z, Ramírez G, Valoyes Z. 2010. Diagnóstico ambiental de las áreas degradadas por la actividad minera en el municipio de Atrato, Chocó. Rev Bioetnia. 7 (1): 23-37. Disponible en: http://iiap.org.co/documents/ d0bd48b33419c17ee30617d63c1b56e7.pdf

Villareal H, Álvarez M, Córdoba S, Escobar F, Fagua G, Gast F, et al. 2004. Manual de métodos para el desarrollo de inventarios de biodiversidad. $2^{\mathrm{a}}$ ed. Bogotá: Programa de Inventarios de Biodiversidad, Instituto de Investigación de Recursos Biológicos Alexander von Humboldt; 236 pp. 


\section{Diversidad de aves en áreas en proceso de regeneración natural. EY Cuesta-Ríos et al.}

Anexo 1

Composición taxonómica y abundancia de la avifauna a áreas en proceso de regeneración natural degradas por minería a cielo abierto de oro y platino en Condoto, Chocó, Colombia

\begin{tabular}{|c|c|c|c|c|c|c|c|}
\hline Orden & Familia & Especies & E1 & E2 & E3 & $\mathbf{N}$ & $\%$ \\
\hline \multirow[t]{2}{*}{ Pelecaniformes } & \multirow[t]{2}{*}{ Ardeidae } & Butorides striata & 0 & 0 & 2 & 2 & 0,30 \\
\hline & & Egretta thula & 0 & 0 & 2 & 2 & 0,30 \\
\hline \multirow[t]{2}{*}{ Accipitriformes } & \multirow[t]{2}{*}{ Accipitridae } & Accipiter superciliosus & 0 & 1 & 0 & 1 & 0,15 \\
\hline & & Accipiter striatus & 0 & 1 & 0 & 1 & 0,15 \\
\hline Charadriiformes & Jacanidae & Jacana jacana & 0 & 0 & 2 & 2 & 0,30 \\
\hline \multirow[t]{5}{*}{ Cuculiformes } & \multirow[t]{5}{*}{ Cuculidae } & Crotophaga ani & 4 & 6 & 12 & 22 & 3,32 \\
\hline & & Crotophaga major & 12 & 13 & 19 & 44 & 6,65 \\
\hline & & Amazilia rosenbergi & 2 & 2 & 0 & 4 & 0,60 \\
\hline & & Amazilia tzacatl & 15 & 17 & 0 & 32 & 4,83 \\
\hline & & Androdon aequatorialis & 1 & 0 & 0 & 1 & 0,15 \\
\hline \multirow[t]{6}{*}{ Apodiformes } & \multirow[t]{6}{*}{ Trochilidae } & Florisuga mellivora & 2 & 0 & 0 & 2 & 0,30 \\
\hline & & Glaucis hirsutus & 4 & 5 & 0 & 9 & 1,36 \\
\hline & & Phaethornis longuemareus & 0 & 1 & 0 & 1 & 0,15 \\
\hline & & Phaethornis syrmatophorus & 2 & 1 & 0 & 3 & 0,45 \\
\hline & & Phaethornis yaruqui & 7 & 6 & 0 & 13 & 1,96 \\
\hline & & Threnetes ruckeri & 0 & 2 & 0 & 2 & 0,30 \\
\hline Trogoniformes & Trogonidae & Trogon chionurus & 0 & 2 & 0 & 2 & 0,30 \\
\hline Coraciiformes & Alcedinidae & Chloroceryle amazona & 0 & 0 & 6 & 6 & 0,91 \\
\hline \multirow[t]{3}{*}{ Piciformes } & \multirow[t]{2}{*}{ Ramphastidae } & Ramphastos brevis & 4 & 0 & 0 & 4 & 0,60 \\
\hline & & Ramphastos swainsonii & 6 & 0 & 0 & 6 & 0,91 \\
\hline & Picidae & Colaptes punctigula & 0 & 1 & 0 & 1 & 0,15 \\
\hline Psittaciformes & Psittacidae & Amazona farinosa & 12 & 12 & 0 & 24 & 3,63 \\
\hline \multirow{9}{*}{ Passeriformes } & \multirow{3}{*}{ Thamnophilidae } & Hylophylax naevioides & 2 & 0 & 0 & 2 & 0,30 \\
\hline & & Myrmotherula axillaris & 4 & 0 & 0 & 4 & 0,60 \\
\hline & & Myrmeciza exsul & 5 & 5 & 0 & 10 & 1,51 \\
\hline & \multirow[t]{6}{*}{ Furnariidae } & Dendrocincla fuliginosa & 1 & 0 & 0 & 1 & 0,15 \\
\hline & & Glyphorynchus spirurus & 1 & 0 & 0 & 1 & 0,15 \\
\hline & & Contopus cinereus & 2 & 0 & 0 & 2 & 0,30 \\
\hline & & Elaenia flavogaster & 16 & 11 & 0 & 27 & 4,08 \\
\hline & & Legatus leucophaius & 2 & 2 & 0 & 4 & 0,60 \\
\hline & & Myiozetetes cayanensis & 0 & 8 & 12 & 20 & 3,02 \\
\hline \multirow[t]{8}{*}{ Tyrannidae } & & Myiozetetes granadensis & 3 & 0 & 0 & 3 & 0,45 \\
\hline & & Myiobius barbatus & 0 & 2 & 0 & 2 & 0,30 \\
\hline & & Pyrocephalus rubianus & 3 & 0 & 0 & 3 & 0,45 \\
\hline & & Tyrannus melancholicus & 12 & 12 & 6 & 30 & 4,53 \\
\hline & & Tyrannus tyrannus & 4 & 0 & 0 & 4 & 0,60 \\
\hline & \multirow[t]{2}{*}{ Pipridae } & Lepidothrix coronata & 7 & 8 & 0 & 15 & 2,27 \\
\hline & & Manacus manacus & 27 & 16 & 0 & 43 & 6,50 \\
\hline & Corvidae & Cyanocorax affinis & 0 & 3 & 0 & 3 & 0,45 \\
\hline
\end{tabular}


Bioetnia Volumen 14, 2017

Anexo 1

Composición taxonómica y abundancia de la avifauna a áreas en proceso de regeneración natural degradas por minería a cielo abierto de oro y platino en Condoto, Chocó, Colombia (continuación)

\begin{tabular}{|c|c|c|c|c|c|c|c|}
\hline Orden & Familia & Especies & E1 & E2 & E3 & $\mathbf{N}$ & $\%$ \\
\hline & \multirow[t]{2}{*}{ Hirundinidae } & Stelgidopteryx ruficollis & 12 & 10 & 0 & 22 & 3,32 \\
\hline & & Hirundo rustica & 10 & 16 & 0 & 26 & 3,93 \\
\hline & \multirow{11}{*}{ Thraupidae } & Mitrospingus casiinii & 2 & 0 & 0 & 2 & 0,30 \\
\hline & & Ramphocelus dimidiatus & 16 & 3 & 3 & 22 & 3,32 \\
\hline & & Ramphocelus flammigerus & 7 & 5 & 0 & 12 & 1,81 \\
\hline & & Saltator grossus & 2 & 0 & 0 & 2 & 0,30 \\
\hline & & Saltator maximus & 6 & 0 & 0 & 6 & 0,91 \\
\hline & & Saltator striatipectus & 2 & 0 & 0 & 2 & 0,30 \\
\hline & & Tachyphonus delatrii & 16 & 31 & 0 & 47 & 7,10 \\
\hline & & Tachyphonus rufus & 2 & 0 & 0 & 2 & 0,30 \\
\hline & & Tangara larvata & 23 & 18 & 0 & 41 & 6,19 \\
\hline & & Thraupis episcupus & 6 & 12 & 1 & 19 & 2,87 \\
\hline & & Thraupis palmarum & 5 & 3 & 3 & 11 & 1,66 \\
\hline & \multirow{3}{*}{ Emberizidae } & Volatina jacarina & 0 & 0 & 23 & 23 & 3,47 \\
\hline & & Sporophila corvina & 0 & 0 & 17 & 17 & 2,57 \\
\hline & & Ozyzoborus funereus & 6 & 6 & 6 & 18 & 2,72 \\
\hline & Cardinalidae & Piranga rubra & 0 & 2 & 0 & 2 & 0,30 \\
\hline & \multirow[t]{2}{*}{ Parulidae } & Parula pitiayumi & 6 & 0 & 0 & 6 & 0,91 \\
\hline & & Parkesia noveboracensis & 2 & 0 & 2 & 4 & 0,60 \\
\hline & \multirow[t]{2}{*}{ Icteridae } & Psarocolius wagleri & 0 & 4 & 0 & 4 & 0,60 \\
\hline & & Molothrus bonariensis & 1 & 3 & 0 & 4 & 0,60 \\
\hline & \multirow[t]{2}{*}{ Frigillidae } & Sporagra xanthogastra & 10 & 0 & 0 & 10 & 1,51 \\
\hline & & Euphonia xanthogaster & 2 & 0 & 0 & 2 & 0,30 \\
\hline & Total & & 296 & 250 & 116 & 662 & 100,00 \\
\hline
\end{tabular}

